\title{
Thermoelectricity in Molecular Junctions
}

Pramod Reddy, ${ }^{1} *$ Sung-Yeon Jang, ${ }^{2,3} * \dagger$ Rachel Segalman, ${ }^{1,2,3} \ddagger$ Arun Majumdar ${ }^{1,3,4} \ddagger$

${ }^{1}$ Applied Science and Technology Program, University of California Berkeley, CA 94720, USA. ${ }^{2}$ Department of Chemical Engineering, University of California, Berkeley, CA 94720, USA. ${ }^{3}$ Materials Science Division, Lawrence Berkeley Laboratory, Berkeley, CA 94720, USA. ${ }^{4}$ Departments of Mechanical Engineering and Materials Science and Engineering, University of California, Berkeley, CA 94720, USA.

*These authors contributed equally to this paper.

†Present address: Optoelectronic Materials Research Center, Korea Institute of Science and Technology, Seoul 136-791, Korea.

¥To whom correspondence should be addressed. E-mail: majumdar@me.berkeley.edu (A.M.); segalman@berkeley.edu (R.S.)

By trapping molecules between two gold electrodes with a temperature difference across them, the junction Seebeck coefficient of 1,4-benzenedithiol (BDT), 4,4'dibenzenedithiol (DBDT) and 4,4"-tribenzenedithiol (TBDT) in contact with gold was measured at room temperature to be $(+8.7 \pm 2.1)$ microvolts per Kelvin $(\mu \mathrm{V} / \mathrm{K}),(+12.9 \pm 2.2) \mu \mathrm{V} / \mathrm{K}$, and $(+14.2 \pm 3.2) \mu \mathrm{V} / \mathrm{K}$, respectively. The positive sign unambiguously indicates ptype (hole) conduction in these heterojunctions, while the Au Fermi level position for Au-BDT-Au junctions was identified to be $1.2 \mathrm{eV}$ above the highest occupied molecular orbital (HOMO) level of BDT. The ability to study thermoelectricity in molecular junctions provides the opportunity to address these fundamental unanswered questions about their electronic structure while also offering an opportunity to begin exploring molecular thermoelectric energy conversion.

Study of charge transport in molecules is of fundamental interest with potential applications in molecular electronics (1) and energy conversion devices (2, 3). Current-voltage (IV) characteristics of single molecules have been extensively investigated by trapping a single molecule in break junctions formed by mechanical strain (4), electromigration (5), and scanning tunneling microscopes (6). While such measurements have provided significant insight about charge transport through molecular junctions, critical aspects about the electronic structure cannot be uniquely obtained by I-V characteristics alone. For example, whether molecular junctions are p-type or n-type, i.e. the position of the Fermi level, $E_{\mathrm{F}}$, of the metal contacts with respect to the highest occupied (HOMO) and lowest unoccupied (LUMO) molecular orbitals, generally remains unknown because of uncertainties in the microscopic details of the contacts (7-10). A specific example of this is benzenedithiol (BDT), in which case the electrical conductance has been studied extensively both experimentally $(4,11)$ and theoretically $(7,12-15)$, but while some groups suggest that the $E_{\mathrm{F}}$ of the electrodes lies close to the HOMO level $(7,13,15)$ other groups contend that $E_{\mathrm{F}}$ lies near the LUMO level $(12,14)$. Although sweeping the gate bias in single-molecule transistors could potentially yield this information, Coulombic interactions caused by charging of the molecule could perturb the electronic structure $(16,17)$.

It has been suggested that the sign of the Seebeck coefficient, $S$, of molecular junctions can indicate the sign of the charge carrier and the relative position of $E_{\mathrm{F}}$ with respect to the HOMO or LUMO levels (8). Indeed, thermopower measurements using a scanning probe microscope have yielded nanoscale spatial distributions of electron and hole concentrations in inorganic semiconductors (18) and have led to chemical potential microscopy at the atomic scale $(19,20)$. Here we report an alternative approach where we measure $S$ for molecular junctions formed by trapping molecules between gold electrodes and measuring the voltage generated across them when a temperature bias is imposed across the junction.

In general $S$ is associated with bulk materials and is obtained by measuring the voltage difference created across a material in response to an applied temperature differential. In such bulk materials, charge transport is diffusive in nature. The concept of an effective $S$ is also valid for junctions where the transport may be ballistic. For such junctions, however, a more general form of the Seebeck coefficient is needed and is given as

$$
S=\frac{1}{e T} \frac{\int_{0}^{\infty} \sigma(E)\left(E-E_{\mathrm{F}}\right) d E}{\int_{0}^{\infty} \sigma(E) d E}
$$


where $\sigma(E)$ is the energy-dependent differential electrical conductivity, $E_{\mathrm{F}}$ is the Fermi level (or more accurately, the chemical potential), $e$ is the charge of an electron, and $T$ is the absolute temperature; the denominator in Eq. 1 is the electrical conductivity, $\sigma$. As Eq. 1 suggests, $S$ reflects the asymmetry of the distribution of conduction electrons or holes with respect to $E_{\mathrm{F}}$. In bulk materials, this asymmetry results from energy-dependent carrier scattering or the asymmetry in the density of states. For ballistic transport, the asymmetry can be created by a potential barrier at a junction, such as that created between $E_{\mathrm{F}}$ of a metal and the HOMO or LUMO level of a molecule. Here, $S$ is not an intrinsic property of a material, but that of the heterojunction. Hence, we call it a junction Seebeck coefficient, $S_{\text {junction }}$. Because $S_{\text {junction }}$ measures the size of an energy barrier, it is not expected to depend on the number of molecules trapped between the electrodes and is, therefore, an intrinsic property of the junction. This is in contrast to the junction's electrical conductance, which depends on the number of molecules.

A modified scanning tunneling microscope (STM) setup is shown schematically in Fig. 1A where a customized control circuit drives a Au STM tip at a constant speed toward a Au substrate in air under ambient conditions. The Au tip is kept in contact with a large thermal reservoir at room temperature, which maintains the tip temperature very close to ambient (21-23). The Au substrate can be heated with an electric heater to a desired temperature above ambient to create a tipsubstrate temperature difference, $\Delta T$. When the Au STM tip approaches the hot substrate, a tip-substrate voltage bias is applied and the current is continuously monitored. When the conductance reaches a sufficiently high threshold of $0.1 G_{o}$, where $G_{o}=2 e^{2} / h$ [see (23) for a discussion on the choice of $0.1 G_{o}$ ] is the quantum of charge conductance, our previous experiments on electrical conductance have shown that the proximity is sufficient to trap molecules between the electrodes (24). Once this threshold is reached, the voltage bias and the current amplifier are disconnected, and the voltage amplifier is connected instead (see Fig. 1A) to measure the tip-substrate thermoelectric voltage induced by $\Delta T$. The tip is then slowly withdrawn to a sufficiently long distance $(\sim 15 \mathrm{~nm})$ and the output voltage $\Delta V$ is continuously monitored with the tip grounded.

When the Au substrate is covered by thiol-terminated molecules, we have shown that molecular bridges are formed between the Au tip and the substrate, and the electrical conductance of single molecules can be monitored in air (24). In our experiment, we covered the Au substrate with benzenedithiol (BDT), dibezenedithiol (DBDT) or tribenzenedithiol (TBDT) (25) molecules. If molecules of BDT, DBDT, or TBDT are trapped between the tip and substrate with a superimposed $\Delta T$, we should expect to see a thermoelectric voltage generated between the electrodes (8), which should last as long as one or more molecules are trapped and vanish once all of the molecules break away. A typical thermoelectric voltage curve obtained in the experiment that was performed with a $\Delta T=20 \mathrm{~K}$ and with the substrate covered with BDT molecules is shown in Fig. 1B. A constant thermoelectric voltage of about $\Delta V=-200 \mu \mathrm{V}$ (blue curve) is observed, which lasts until all of the molecules trapped in the junction break away. Note that the distance the tip travels ( 1 to $2 \mathrm{~nm}$ ) before the molecule breaks away is much longer the molecule length. Because the thiol group on the molecule binds sufficiently strongly to $\mathrm{Au}$, and because $\mathrm{Au}$ atoms are sufficiently mobile at room temperature, it has been proposed that Au chains are formed both on the tip and the substrate when the STM tip is pulled away (6). In contrast to electrical conductance measurements (24) where steps are seen in the electrical conductance as molecules break away one at a time, no steps are seen in the $\Delta V$, which suggests that $S_{\text {junction }}$ is independent of the number of molecules. As the $\Delta T$ is increased from $0 \mathrm{~K}$ to $30 \mathrm{~K}$, the thermoelectric voltage signal increases (Fig. 1C). Control experiments performed on clean gold surfaces without any molecules, as shown in Figure 1B (red), demonstrate that no measurable $\Delta V$ is generated in the absence of molecules.

To obtain a statistically significant value of $\Delta V$ of a AuBDT-Au junction, roughly 1000 consecutive experiments were performed at each value of $\Delta T$. These data were used to construct histograms for each temperature differential without any data pre-selection. The histograms thus obtained are shown in Fig. 2, A to C, which were used to estimate the average and the variation in $S_{\text {junction }}$. The relation between $S_{\text {junction }}$ of the Au-molecule-Au junction and the measured voltage is (23):

$$
S_{\text {junction }}=S_{\mathrm{Au}}-\frac{\Delta V}{\Delta T}
$$

where $S_{\mathrm{Au}}$ is the Seebeck coefficient of bulk Au, which is $1.94 \mu \mathrm{V} / \mathrm{K}$ at $300 \mathrm{~K}$ (26). In Fig. $2 \mathrm{D} \Delta V_{\text {peak }}$ is plotted as a function of $\Delta T$, where $\Delta V_{\text {peak }}$ corresponds to the $\Delta V$ at the peak of the distribution. The error bars in Fig. 2D correspond to the full-width half-maximum of the distributions. From the slope $\Delta V_{\text {peak }} / \Delta T$ and Eq. 1, one obtains $S_{\text {Au-BDT-Au }}=+8.7 \pm 2.1$ $\mu \mathrm{V} / \mathrm{K}$. Similar experiments were also performed with DBDT and TBDT and statistical analysis reveals that $S_{\text {Au-DBDT-Au }}=$ $+12.9 \pm 2.2 \mu \mathrm{V} / \mathrm{K}$ and $S_{\text {Au-TBDT-Au }}=+14.2 \pm 3.2 \mu \mathrm{V} / \mathrm{K}$ (23), as displayed in Fig. 2E. There seems to be a linear dependence of thermopower with molecule length, which is in contrast to the exponential dependence of electrical resistance that is generally attributed to tunneling across the molecule. The histograms of $\Delta V$ for Au-TBDT-Au junctions at $20 \mathrm{~K}$ and 30 $\mathrm{K}$, [fig. S4, A to C (23)] exhibit deviations from a Gaussian curve. Further, the full-width at half-maxima for the histograms at $20 \mathrm{~K}$ and $30 \mathrm{~K}$ increased considerably 
compared to that of Au-BDT-Au junctions, which may arise from the effect of the conformational changes in the molecules trapped in the junctions. The plot of the peak values of the histograms versus the temperature differential for DBDT and TBDT [figs. S3D and S4D (23)] show deviations from linearity. This deviation may arise because the applied temperature differentials of 20 and $30 \mathrm{~K}$ across molecules are sufficiently high such that linear transport theory may be inadequate to completely describe the temperature dependence of the thermoelectric voltage.

The relative position of the HOMO and LUMO levels with respect to $E_{\mathrm{F}}$, of the metal electrodes can be related to the measured value of $S_{\text {junction }}(8)$. The Landauer formula (27) is used to relate $S_{\text {junction }}$ to the transmission function, $\tau(E)$. It is shown that $S_{\text {junction }}$ can be obtained as:

$$
S_{\text {junction }}=-\left.\frac{\pi^{2} k_{B}^{2} T}{3 e} \frac{\partial \ln (\tau(E))}{\partial E}\right|_{E=E_{\mathrm{F}}}
$$

The transmission function for the case of Au-BDT-Au junction, that was derived using the non-equilibrium Green's function formalism in conjunction with extended Huckel theory (8), is shown in Fig. 3A. It is clear that $\tau(E) \sim 1$ when the $E_{\mathrm{F}}$ aligns with either the HOMO or the LUMO levels, and decreases rapidly to below 0.01 in between. Using this transmission function in Eq. 3, we calculate $S_{\text {junction }}$ (Fig. 3B) and find that $S_{\text {junction }}$ is positive (p-type) if $E_{\mathrm{F}}$ is closer to the HOMO level and negative (n-type) if it closer to the LUMO level. Using the measured value of $S_{\text {Au-BDT-Au }}=+8.7 \pm 2.1$ $\mu \mathrm{V} / \mathrm{K}$, we can see from Fig. $3 \mathrm{~B}$ that $E_{\mathrm{F}}$ is $\sim 1.2 \mathrm{eV}$ from the HOMO level. The value of the transmission function at this relative position of the Fermi level can be seen to be $\tau(E) \sim$ 0.01 from Fig. 3A. In the Landauer formalism, we know that the conductance $G_{\text {molecule }}$ can be related to the transmission function at $E_{\mathrm{F}}$ as:

$$
\left.G_{\text {molecule }} \approx \frac{2 e^{2}}{h} \tau(E)\right|_{E=E_{\mathrm{F}}}=\left.\tau(E)\right|_{E=E_{\mathrm{F}}} G_{o} \text { (4) }
$$

Eq. 4 would imply that the conductance of BDT should be $\sim 0.01 G_{o}$. This estimated value of the electrical conductance is in excellent agreement with the measured electrical conductance BDT $(11,28)$.

Junction Seebeck coefficient measurements can provide insight about the electronic structure of the heterojunction, but the results also bear on an as yet unexplored field of thermoelectric energy conversion based on molecules. The best efficiency in thermoelectric energy conversion can be achieved if charge transport through occurs through a single energy level (29, 30). Single-level transport is, however, difficult to realize in inorganic materials. Metal-moleculemetal heterojunctions are ideal in this regard since they (i) provide transport either through the HOMO or LUMO levels and (ii) have very low vibrational heat conductance because of large mismatch of vibrational spectra between the bulk metal and discrete molecules (31). Hence, such a hybrid material offers the promise of efficient thermoelectric energy conversion devices. What have remained unknown so far are the values of the Seebeck coefficients of such molecular junctions and the ways to change the junction Seebeck coefficient and electrical conductance. The length dependence of molecular junction Seebeck coefficients is shown in Fig. 2E for the molecules we have studied, but there may be other ways of tuning thermopower, such as by introducing various chemical moieties in the molecule or by controlling the metal-molecule chemical bond.

\section{References and Notes}

1. A. Aviram, M. A. Ratner, Chem. Phys. Lett. 29, 277 (1974).

2. W. U. Huynh, J. J. Dittmer, A. P. Alivisatos, Science 295, 2425 (2002).

3. G. Yu, J. Gao, J. C. Hummlen, F. Wudl, A. J. Heeger, Science 270, 1789 (1995).

4. M. A. Reed, C. Zhou, C. J. Muller, T. P. Burgin, J. M. Tour, Science 278, 252 (1997).

5. H. Park et al., Nature 407, 57 (2000).

6. B. Q. Xu, N. J. J. Tao, Science 301, 1221 (2003).

7. P. Damle, A. W. Ghosh, S. Datta, Chem. Phys. 281, 171 (2002).

8. M. Paulsson, S. Datta, Phys. Rev. B. 67, 241403R (2003).

9. F. Zahid, A. W. Ghosh, M. Paulsson, E. Polizzi, S. Datta, Phys. Rev. B. 70, 245317 (2004).

10. Consider a molecule strongly bound to one electrode and very weakly bound to the other. An example of this is scanning tunneling spectroscopy, where the tip is weakly interacting with the molecule, which is strongly bound to a substrate. Under bias, such a configuration produces asymmetric I-V curves because the chemical potential of the tip crosses the HOMO and LUMO levels of the molecule, while that of the substrate is pinned to the molecular levels. Hence, by applying a tip bias, one can determine the chemical potential position with respect to the HOMO and LUMO levels. However, to make realistic molecular devices, the molecule cannot be weakly bound to one electrode, since otherwise the device would be mechanically unstable and electrically irreproducible due to uncertainties of the contact. But in the case of a molecule strongly bound to both the electrodes, the chemical potential of both electrodes is pinned and hence, symmetric I-V curves are produced under bias (9). This symmetry in the I-V characteristics makes it impossible to determine whether transport is through HOMO or LUMO levels (9).

11. X. Y. Xiao, B. Q. Xu, N. J. Tao, Nano. Lett. 4, 267 (2004). 
12. M. Di Ventra, S. T. Pantelides, N. D. Lang, Phys. Rev. Lett. 84, 979 (2000).

13. E. G. Emberly, G. Kirczenow, Phys. Rev. B. 58, 10911 (1998).

14. J. G. Kushmerick et al., Phys. Rev. Lett. 89, 086802 (2002).

15. J. Taylor, M. Brandbyge, K. Stokbro, Phys. Rev. Lett. 89, 138301 (2002).

16. W. J. Liang, M. P. Shores, M. Bockrath, J. R. Long, H. Park, Nature 417, 725 (2002).

17. J. Park et al., Nature 417, 722 (2002).

18. H. K. Lyeo et al., Science 303, 816 (2004).

19. C. C. Williams, H. K. Wickramasinghe, Nature 344, 317 (1990).

20. J. C. Poler, R. M. Zimmerman, E. C. Cox, Langmuir 11, 2689 (1995).

21. One might suspect that the heat flow from the substrate to the tip may cause the tip temperature to increase. However, tip-substrate heat transport is mostly due to conduction through air (thermal conductance through the molecules trapped in between the tip and the substrate or through a liquid meniscus if present is very small in comparison) (22). Since, the tip-sample thermal resistance is sufficiently larger than that between the Au tip and the thermal reservoir, the Au tip will be at the reservoir temperature. This has been shown to be true in previous work by our group $(22,23)$ (further analysis that supports the idea that the tip is at ambient temperature is provided in supplementary information). Hence, the temperature difference applied across the substrate and the reservoir is the same as that across the tip-substrate junction.

22. L. Shi, A. Majumdar, J. Heat. Trans. 124, 329 (2002).

23. Additional experimental details, data, and analysis are available at Science online.

24. S. Y. Jang, P. Reddy, A. Majumdar, R. A. Segalman, Nano. Lett. 6, 2362 (2006).

25. B. de Boer et al., Langmuir 19, 4272 (2003).

26. F. J. Blatt, Thermoelectric power of metals (Plenum Press, New York, 1976), pp. xv, 264.

27. M. Buttiker, Y. Imry, R. Landauer, S. Pinhas, Phys. Rev. B 31, 6207 (1985).

28. Note that Paulsson and Datta (8) used the measurements of Poler et al. (20) for an asymmetric junction to predict the position of $E_{\mathrm{F}}$ with respect to HOMO/LUMO levels of a monolayer.

29. G. D. Mahan, J. O. Sofo, Proc. Nat. Acad. Sci. 93, 7436 (1996).

30. T. E. Humphrey, H. Linke, Phys. Rev. Lett. 94, 096601 (2005).

31. R. Y. Wang, R. A. Segalman, A. Majumdar, Appl. Phys. Lett. 89,173113 (2006).
32. We gratefully acknowledge support from the National Science Foundation under Grant No. EEC-0425914 and the NSF-NSEC COINS, the Berkeley-ITRI Research Center, and the Department of Energy Basic Energy Sciences (DOE-BES) Thermoelectrics Program and DOEBES Plastic Electronics Program at the Lawrence Berkeley National Laboratory.

\section{Supporting Online Material}

www.sciencemag.org/cgi/content/full/1137149/DC1

Materials and Methods

Figs. S1 to S6

References

3 November 2006; accepted 29 January 2007

Published online 15 February 2007; 10.1126/science.1137149

Include this information when citing this paper.

Fig. 1. Experimental setup and measurements. (A) Schematic description of the experimental set up based on a scanning tunneling microscope (STM) break junction. Molecules of benzenedithiol (BDT), dibenzenedithiol (DBDT), or tribenzenedithiol (TBDT) are trapped between the Au STM tip kept at ambient temperature and a heated Au substrate kept at temperature $\Delta T$ above the ambient. When the tip approaches the substrate, a voltage bias is applied and the current is monitored to estimate the conductance. When the conductance reaches a threshold of $0.1 G_{o}$, the voltage bias and the current amplifier are disconnected. A voltage amplifier is then used to measure the induced thermoelectric voltage, $\Delta V$, while the tip is gradually pulled away from the substrate. (B) A plot of the thermoelectric voltage measured as a function of the tip-sample distance when a temperature differential $\Delta T=20 \mathrm{~K}$ is applied (Au tip at ambient and substrate at ambient $+20 \mathrm{~K})$. The blue curve is obtained when a Au-benzenedithiol-Au junction is broken. The red curve shows a control experiment performed on a clean gold substrate; (C) Typical thermoelectric voltage traces for tipsubstrate temperature differentials of $0 \mathrm{~K}, 10 \mathrm{~K}, 20 \mathrm{~K}$, and 30 $\mathrm{K}$ for Au-BDT-Au junctions.

Fig. 2. Histograms obtained by analyzing approximately 1000 consecutive thermoelectric voltage curves obtained in measurements of Au-BDT-Au junctions. (A) with tipsubstrate temperature differential $\Delta T=10 \mathrm{~K}$; (B) $\Delta T=20 \mathrm{~K}$; (C) $\Delta T=10 \mathrm{~K}$. (D) Plot of the peak values of the thermoelectric voltage in histograms as a function of the temperature differential. The error bars represent full width half maximum of the corresponding histograms. It can be seen that the measured voltage varies linearly with the temperature differential as expected. (E) Plot of measured junction Seebeck coefficient as a function of molecular length for BDT, DBDT and TBDT. 
Fig. 3. Relating the measured Seebeck coefficient of $\mathrm{Au}-$ BDT-Au junction to the position of Fermi level. (A) Theoretical prediction (8) of the transmission function of a Au-BDT-Au junction plotted as a function of the relative position of the Fermi level of the Au electrodes with respect to the HOMO and LUMO levels. (B) The predicted (8) Seebeck coefficient of a Au-BDT-Au junction as a function of the relative position of the Fermi level with respect to the HOMO and LUMO levels. When the measured value of $S_{\mathrm{Au}-}$ BDT-Au $=+8.7 \pm 2.1 \mu \mathrm{V} / \mathrm{K}$ (blue band) is used in this figure, it is clear that the Fermi level is $\sim 1.2 \mathrm{eV}$ above the HOMO

level. At this energy level, the transmission function is $\tau(E) \sim$ 0.01 . 
A
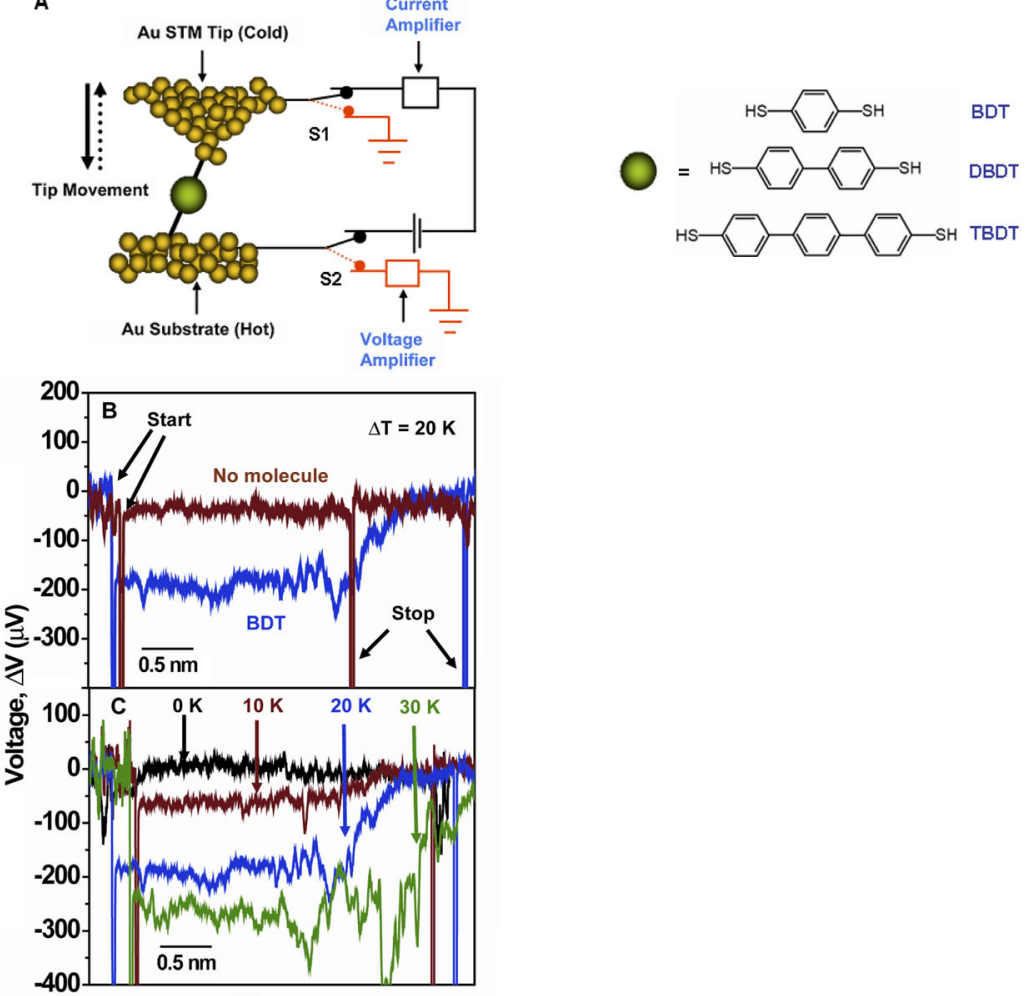

Distance 


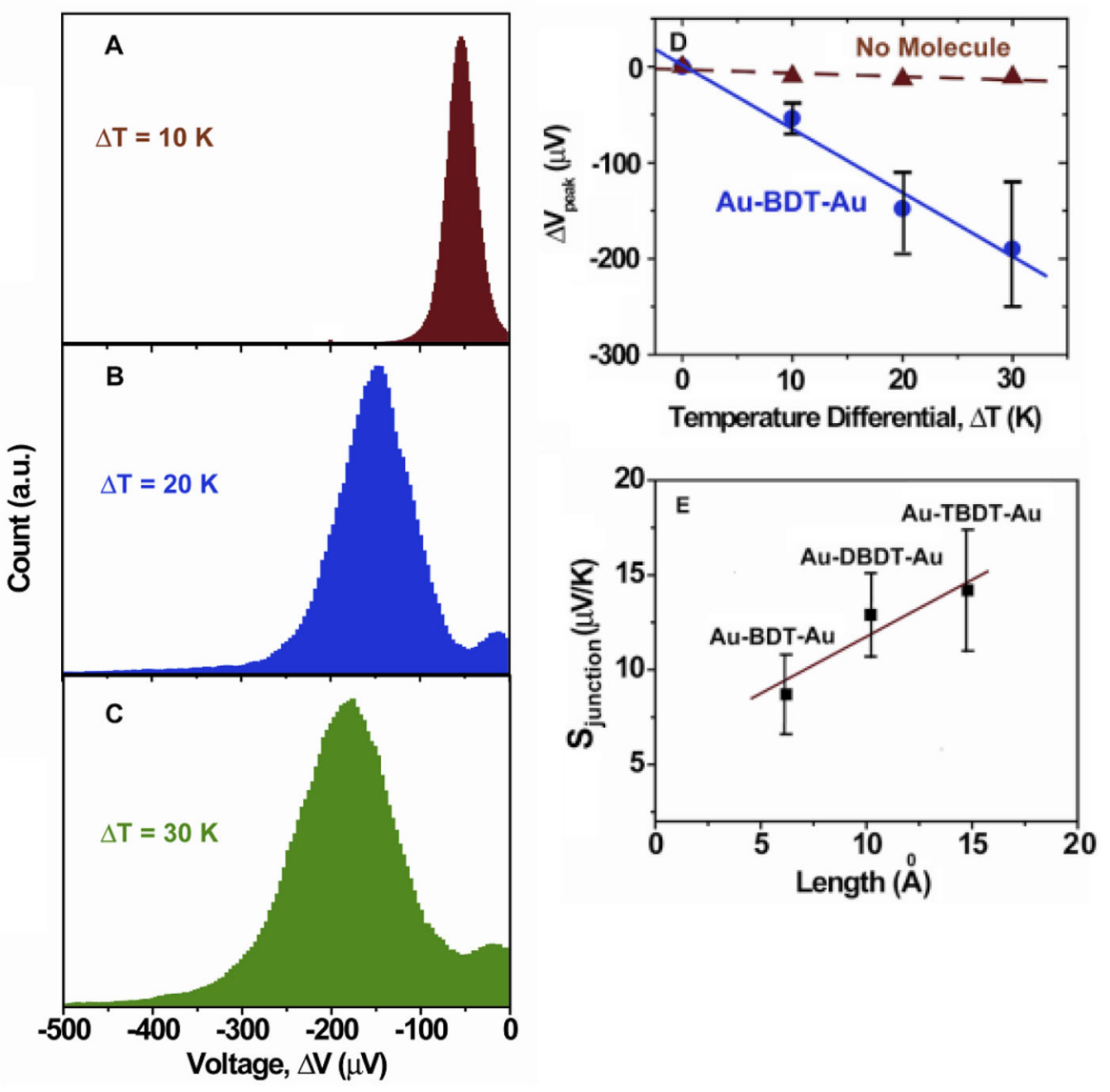




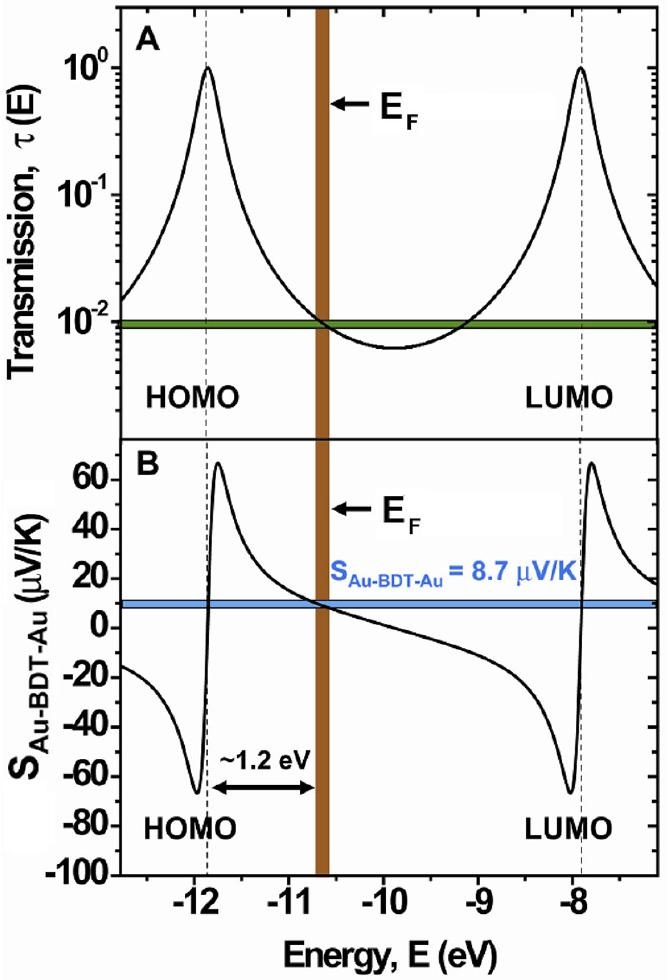

